\title{
SALL4 is essential for cancer cell proliferation and is overexpressed at early clinical stages in breast cancer
}

\author{
DAISUKE KOBAYASHI, KAGEAKI KURIBAYSHI, MAKI TANAKA and NAOKI WATANABE
}

\begin{abstract}
Department of Clinical Laboratory Medicine, Sapporo Medical University School of Medicine, Sapporo, Japan
\end{abstract}
Received September 29, 2010; Accepted November 24, 2010

DOI: $10.3892 /$ ijo.2011.929

\begin{abstract}
Few target molecules have been identified that enable the diagnosis of breast cancer with a high sensitivity and specificity, especially in the early clinical stages of cancer. Here, we present the first evidence for diagnostic performance of gene expression for SALL4, a transcription factor that plays an essential role in the embryonic development and self-renewal of embryonic stem (ES) cells, in breast cancer. The sensitivity and specificity of SALL4 was 80.4 and $80.0 \%$, respectively, as estimated using the cut-off value obtained from the analysis of the receiver operating characteristic curve. Furthermore, comparison of paired cancer and non-cancer tissues from the same breast cancer patient revealed elevated SALL4 mRNA levels in $86.1 \%$ $(31 / 36)$ of the specimens. No obvious correlations were detected between clinicopathological factors and SALL4 mRNA expression; however, SALL4 mRNA was expressed at a high level even in the early clinical stages of the cancer. An siRNA experiment to determine the significance of SALLA expression showed complete inhibition of proliferation in breast cancer MCF7 cells. This inhibitory effect of siRNA was induced by cell cycle arrest mainly at the G1 phase, leading to increased cell volume. These results suggest that SALL4 mRNA may be a new tool to support the diagnosis of breast cancer, and it may also represent a novel therapeutic target.
\end{abstract}

\section{Introduction}

To increase the efficacy of breast cancer therapy, it is important to identify candidate genes that are essential in cancer cell proliferation and would facilitate tumor detection at high sensitivity and specificity rates, even in the early stages of the disease (1). Measurement of gene expression of such molecules by highly sensitive amplification method would be useful to support the diagnosis, especially when very few

Correspondence to: Dr Naoki Watanabe, Department of Clinical Laboratory Medicine, Sapporo Medical University School of Medicine, South-1, West-16, Chuo-Ku, Sapporo 060-8543, Japan

E-mail:watanabn@sapmed.ac.jp

Key words: SALL4, mRNA expression, early breast cancer, siRNA, cell cycle cells can be obtained and malignancy cannot be definitively diagnosed by histological or cytological methods. Previous studies have shown that some molecules could be useful to distinguish cancer samples from non-cancer samples by measuring expression of single or multiple genes in tissue, peripheral blood cells, or serum (2-4). However, in actual use as a marker to support the diagnosis, there has been a limit in the specificity and sensitivity because of non-negligible level of expression in non-cancer samples or low detection rate in cancer samples even with high specificity.

Cancer cells possess the characteristic ability of selfrenewal via uncontrollable proliferation and dedifferentiation, similar to embryonic stem (ES) cells (5). Detection of the expression of molecules that are crucial to the self-renewal of ES cells might reveal essential aspects of carcinogenesis. Thus, if the expression of these molecules is upregulated in cancers, these molecules represent ideal candidates to support the diagnosis of cancers. Some molecules such as Nanog, Klf4, Oct3/4, and Sall4, which are expressed during early embryonic development, play important roles in the maintenance of self-renewal in mouse ES cells (6-9). These molecules also generate and maintain the self-renewal of induced pluripotent stem (iPS) cells in mice and humans (10-12). Nanog and Sall4 are key factors for the maintenance of undifferentiated state and cell proliferation, respectively $(13,14)$. Knockdown of Sall4 expression leads to catastrophic ES cell proliferation, and Sall4 knockout mice do not survive from embryonic day 7 (14).

Sall4 is the mouse homolog of the Drosophila homeotic gene spalt (sal) and is required for the early development of the posterior head and anterior tail of Drosophila (15). Sal also regulates pattern formation and cell fate decisions in the wing disc, trachea, and sensory organs. Mutations in the human homolog SALLA are known to cause Okihiro syndrome (Duane-radial ray syndrome) characterized by limb deformities and loss of eye movement $(16,17)$. In some cases, anomalies of the rectum, ear, heart, and kidney are observed.

The SALL4/Sall4 gene is constitutively expressed in CD34positive hematopoietic stem cells of humans and mice (18). Interestingly, the overexpression of the Sall4 gene leads to leukemogenesis by increased leukemic cells with markers for stem cells in $50 \%$ of transgenic mice (18). In fact, this gene is overexpressed in various types of human hematopoietic malignancies such as acute myelocytic and lymphocytic leukemia $(19,20)$. SALL4 upregulates the expression of the oncogene Bmi-1 in human hematopoietic stem cells 
and leukemic cells (21). Bmi-1 activates telomerase reverse transcriptase and thereby induces telomerase activity, leading to the transformation of human non-cancer epithelial cells (22). Bmi-1 also inhibits the function of INK4a/ARF, usually disturbing cyclin-dependent kinases 2, 4, and 6 (23), indicating that Bmi-1 expression leads to the progression of the cell cycle from the G1 to the S-phase. These observations strongly suggest the oncogenic potential of SALL4, allowing us to speculate that SALL4 might be overexpressed and potentially used as a diagnostic marker in human malignancies. With regard to this aspect, Cao et al, reported the use of SALL4 protein expression as a novel diagnostic marker for germ cell tumors of the testis and ovary, and metastatic germ cell tumors (24-26). However, there have been no studies that assess the diagnostic ability of SALL4 in non-germ cell tumors. In cases in which a definite pathologic diagnosis cannot be obtained because of the limited cell number acquired using needle or aspiration biopsies, a gene amplification technique should be ideal from the viewpoint of detection sensitivity when assessing the clinical use of this marker to support diagnosis. Therefore, in this study, we determined the mRNA expression of SALL4 in various tissues from breast cancer patients. By using siRNA against the SALL4 gene, we also determined the significance of SALL4 expression in breast cancer cells

\section{Patients and methods}

Patients and frozen tissue samples. Cancer $(\mathrm{n}=51)$ and noncancer $(n=40)$ tissue specimens were obtained from breast cancer patients after surgery. Paired cancer specimens along with the adjacent non-cancer tissue samples were able to obtain from 36 patients. After informed consent was obtained, samples were dissected from these tissue specimens and immediately frozen in liquid nitrogen in a screw-capped cryotube. The tissue samples were stained with hematoxylin/ eosin and reviewed by experienced pathologists. Clinicopathological factors and clinical stages were evaluated according to the criteria of the Japanese Society of Breast Cancer, which are based on the tumor-node-metastasis (TNM) staging system.

Cell culture. The human breast cancer cell lines MCF7 and T47D were obtained from the American Type Culture Collection (Manassas, VA). MCF7 and T47D cells were cultured in Eagle's minimum essential medium (BioWhittaker, Walkersville, MD) and RPMI-1640 (BioWhittaker) supplemented with $10 \%$ heat-inactivated fetal bovine serum (FBS; Invitrogen, Carlsbad, $\mathrm{CA}$ ) at $37^{\circ} \mathrm{C}$ in a humidified atmosphere of $5 \% \mathrm{CO}_{2}$.

Quantification of SALL4 mRNA expression. The expression of SALL4 mRNA was determined by performing quantitative reverse transcriptase-polymerase chain reaction (RT-PCR) on an ABI PRISM 7700 sequence detection system (Applied Biosystems, Foster City, CA). Total RNA was isolated using the Isogen reagent (Nippon Gene, Toyama, Japan), according to the manufacturer's instructions. The RNA concentration was determined using the GeneQuant DNA/RNA Calculator (Amersham Pharmacia Biotech, Uppsala, Sweden). The intron- spanning, gene-specific primers and fluorescent hybridization probes used in the quantitative PCR were as follows: SALL4 forward primer, 5'-AAG TGT AAG GGT CGG AGC AGT CT-3'; reverse primer, 5'-AAT GTC GAG GGT CCC ACA AA-3'; and probe, 5'-(FAM) CCA CGT TTA TCC GAG CCC CGC (TAMRA)-3'. Quantitative RT-PCR was performed using the TaqMan One-Step RT-PCR Master Mix Reagent Kit (Applied Biosystems). To compare SALL4 mRNA expression in different samples, its levels were normalized to the levels of $18 \mathrm{~S}$ ribosomal RNA (rRNA). The expression of 18S rRNA was determined using the TaqMan Ribosomal RNA Control Reagent Kit (Applied Biosystems) according to the manufacturer's instructions. For each experiment, a calibration curve using control RNA from MKN45 cells was prepared. Briefly, by using a computer algorithm, reporter dye emission and quenching dye emission were analyzed during PCR amplification, and the intensity of fluorescent signals during each PCR cycle was detected. Amplification curves obtained from serial dilutions of control RNA were prepared, and the optimal signal intensity (threshold) was manually selected in the exponential phase of the curves. Finally, each PCR cycle number (threshold cycle, $\mathrm{C}_{\mathrm{T}}$ ) at each concentration of initial RNA was determined to draw a calibration curve. The calibration curve was established as an $x y$ plot (the log of the input amount (log ng of initial total RNA) as $x$ and $\mathrm{C}_{\mathrm{T}}$ as $y$ ). The expression levels of the target mRNA in the unknown samples were determined from the $\mathrm{C}_{\mathrm{T}}$ value. A control mix that lacked a template was included in each experiment.

Transduction of small inhibitory RNA (siRNA) against SALL4. HP GenomeWide siRNA, an siRNA designed to target the coding region (exon 2) of the SALL4 gene (GenBank accession no. NM-020436), was obtained from Qiagen (Tokyo, Japan). Single-strand RNAs were annealed by incubating each strand in the siRNA suspension buffer at $90^{\circ} \mathrm{C}$ for $1 \mathrm{~min}$ and then at $37^{\circ} \mathrm{C}$ for $1 \mathrm{~h}$. Non-silencing control RNA (NSC, Qiagen) was used as a transduction control. The transduction of siRNA was performed using the Nucleofector II device and the Cell Line Nucleofector Kit V (Amaxa Inc., Gaithersburg, MD) according to the manufacturer's instructions.

In brief, $\sim 2-4 \times 10^{6}$ cells were cultured under normal conditions to subconfluency, and $1 \times 10^{6}$ cells were transduced with siRNA or NSC in a cuvette. Next, $5 \times 10^{4}$ of these transduced cells were plated in $3 \mathrm{ml}$ of medium supplemented with $10 \%$ FBS in a 6-well plate (Costar, Tokyo, Japan). After $48 \mathrm{~h}$, the expression of the silenced mRNA was quantified by TaqMan RT-PCR. The cells were collected at different periods $(24,48,72$, and $96 \mathrm{~h})$ and subjected to cell number count and cell cycle analysis. Prior to the assay, the cells in the 6-well culture plate were photographed using the BZ-8100 fluorescent microscope (Keyence Co., Ltd, Osaka, Japan), immediately after removal from the incubator, and the digital image was acquired.

Cell cycle analysis. Cells plated onto 6-well culture dishes (Costar) were trypsinized and washed with FBS-free media and PBS. The cells were treated with $1 \mathrm{ml}$ of hypotonic fluorochrome solution $(50 \mu \mathrm{g} / \mathrm{ml}$ propidium iodide, $0.1 \%$ sodium citrate, $0.1 \%$ Triton $\mathrm{X}-100)$ at room temperature for $30 \mathrm{~min}$. The cells were then kept on ice, and 20,000 cells/ 


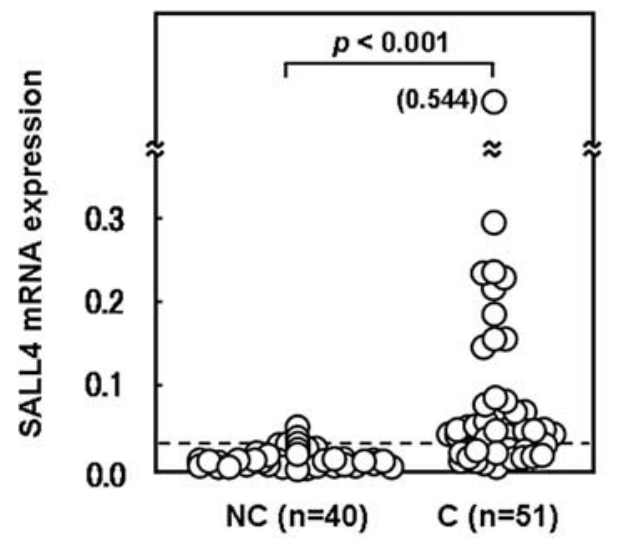

Figure 1. SALL4 mRNA expression in breast cancer and non-cancer tissues. SALL4 mRNA expression level was measured by quantitative RT-PCR and is reported relative to the amount of $18 \mathrm{~S}$ rRNA. The dotted line shows the mean +2 SDs of SALL4 mRNA expression levels in non-cancer tissues. NC, non-cancer tissues; $\mathrm{C}$, cancer tissues. Statistical analysis was performed using the Mann-Whitney rank-sum test. ${ }^{*} \mathrm{p}<0.001$.

sample were analyzed on the EPICS XL flow cytometer (Beckman Coulter, Inc., Fullerton, CA).

Statistical analysis. Statistical analysis of the differences in the expression levels of SALL4 mRNA were analyzed using the Mann-Whitney rank-sum test. Statistical significance was defined as $\mathrm{p}<0.05$. The receiver operating characteristics (ROC) curve was generated, and the sensitivity/specificity at various cutoff values was calculated using StatFlex software version 5.0 (Artech, Osaka, Japan). The software automatically arranges the data that are manually inputted for both groups (non-cancer and cancer tissues) according to the magnitude of the values. It then calculates the sensitivity and specificity at each cutoff value that is determined for each data set and automatically generates the ROC curve.

\section{Results}

Expression of SALL4 mRNA in breast cancers. Quantitative RT-PCR was performed on non-cancer and cancer tissue samples obtained from patients with breast cancer (Fig. 1). The mean SALL4 mRNA expression level in cancer tissues $(0.083 \pm 0.101)$ was remarkably higher than that in non-cancer tissues $(0.013 \pm 0.011)(\mathrm{p}<0.001)$. The cutoff value for $S A L L 4$ mRNA expression was set as the mean \pm 2 standard deviations (SDs) (0.035) of its expression in non-cancer breast tissue. SALL4 mRNA expression was found to be positive in $74.5 \%$ of the cancer tissues (38 of 51) and $5.0 \%$ of the non-cancer tissues (2 of 40) (Fig. 1). The sensitivity and specificity of SALL4 mRNA detection by using the ROC curve was 80.4 and $80.0 \%$, respectively, at a cutoff value for which the difference between sensitivity and specificity was minimal (0.019; Fig. 2). In this analysis, a relatively low sensitivity $(67.4 \%)$ and high specificity $(95.0 \%)$ was obtained at a cutoff value (0.031) close to the mean \pm 2 SDs of the expression level in non-cancer tissues. Furthermore, SALL4 mRNA expression was elevated $>2$-fold in $86.1 \%$ (31/36) of cancer specimens that were obtained along with the adjacent noncancer tissue samples (Fig. 3). The remaining 5 specimens

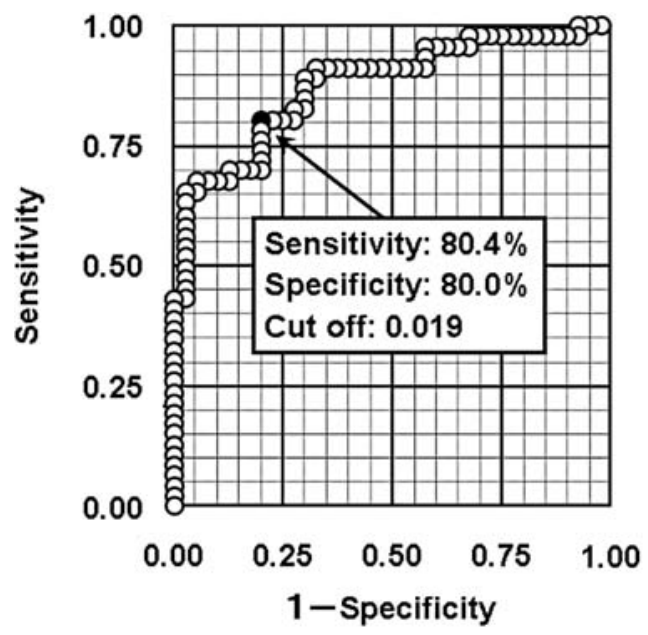

Figure 2. ROC curve for the detection of breast cancer. The curve was automatically generated from multiple points representing various cutoff values determined by StatFlex, version 5.0.

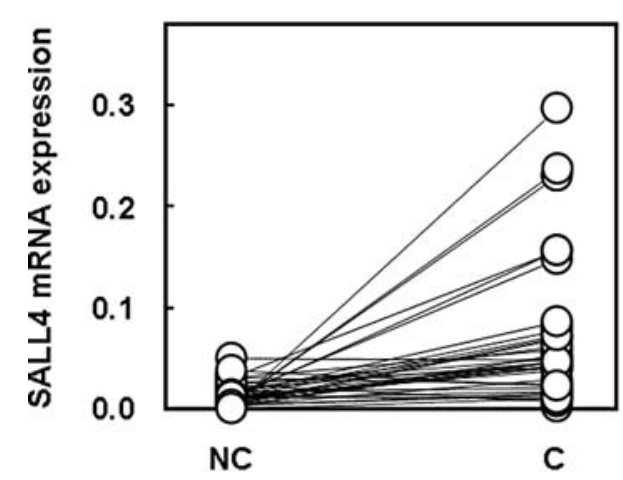

Figure 3. Comparison of the expression levels of SALL4 mRNA between cancer tissues and paired non-cancer tissues. SALL4 mRNA expression level is reported relative to the amount of $18 \mathrm{~S}$ rRNA. The solid line indicates paired samples.

showed an increase of $<2$-fold or a slight decrease in SALL4 mRNA expression as compared to non-cancer tissues.

We then analyzed the relationships between the clinicopathological factors and SALL4 mRNA expression in cancer tissues (Table I). No statistically significant correlations of SALL4 mRNA expression were found with menopausal status, lymph node metastasis, and presence of estrogen or progesterone receptors. Interestingly, the samples of scirrhous carcinoma and premenopausal state showed $>2$-fold higher expression of SALL4 mRNA than those of papillotubular carcinoma and postmenopausal state, but statistical significance $(\mathrm{p}<0.05)$ was obtained only in the analysis for tissue types.

Analysis of the clinical stages of breast cancer, as determined by tumor size, invasion, and metastasis to lymph nodes and distant organs, showed that the mean value and range of SDs in stage I and IIA cancers $(0.074 \pm 0.109)$ were almost equal to those in the other clinical stages (stages IIB, III, and IV; $0.089 \pm 0.089 ; \mathrm{p}>0.05$ ) (Fig. 4). There was no significant difference in the positivity rate between the 2 groups when they were evaluated by the cutoff value $(0.019)$ from 
Table I. Relationship between various clinicopathological factors and SALL4 mRNA expression.

\begin{tabular}{|c|c|c|}
\hline Background & Mean \pm SD & p-value \\
\hline \multicolumn{3}{|l|}{ Tissue type } \\
\hline Papillotubular (n=27) & $0.056 \pm 0.070$ & \\
\hline Scirrhous $(n=14)$ & $0.129 \pm 0.139$ & $<0.05$ \\
\hline Others $(\mathrm{n}=7)$ & $0.081 \pm 0.089$ & $\mathrm{NC}^{\mathrm{a}}$ \\
\hline \multicolumn{3}{|l|}{ Menopausal status } \\
\hline Premenopausal $(n=13)$ & $0.134 \pm 0.156$ & \\
\hline Postmenopausal $(n=35)$ & $0.060 \pm 0.061$ & $>0.05$ \\
\hline \multicolumn{3}{|l|}{ Lymph node metastasis } \\
\hline Negative $(n=25)$ & $0.065 \pm 0.079$ & \\
\hline Positive $(n=23)$ & $0.106 \pm 0.122$ & $>0.05$ \\
\hline \multicolumn{3}{|l|}{ Estrogen receptor } \\
\hline Negative $(n=24)$ & $0.077 \pm 0.120$ & \\
\hline Positive (n=23) & $0.087 \pm 0.079$ & $>0.05$ \\
\hline \multicolumn{3}{|l|}{ Progesterone receptor } \\
\hline Negative $(n=23)$ & $0.058 \pm 0.062$ & \\
\hline Positive $(n=20)$ & $0.110 \pm 0.129$ & $>0.05$ \\
\hline
\end{tabular}

${ }^{a} \mathrm{NC}$, could not be calculated because of small sample numbers.

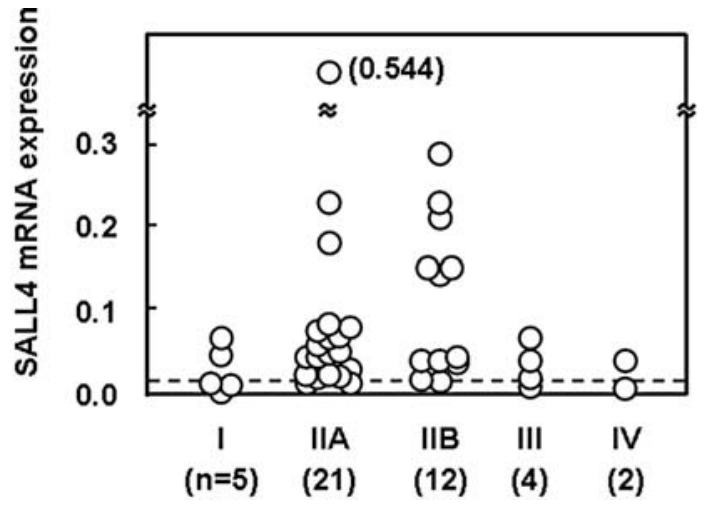

Figure 4. SALL4 mRNA expression in each clinical stage of breast cancer SALL4 mRNA level was measured by quantitative RT-PCR and expressed relative to the amount of $18 \mathrm{~S}$ rRNA. The dotted line shows the cutoff value (0.019) obtained from ROC analysis.

ROC analysis [stage I and IIA, 80.7\% (21/26) vs. stage IIB-IV, $77.8 \%$ (14/18)]. Overall, high SALL4 expression levels were observed even in the relatively early stages of breast cancer.

Effect of SALL4 siRNA transduction on cell proliferation and cell cycle. To determine the role of SALL4 expression in cancer cells, we transduced SALLA siRNA into breast cancer MCF7 cells. Transduction of siRNA decreased the SALL4 mRNA expression level to $28 \%$ of the baseline levels after
A

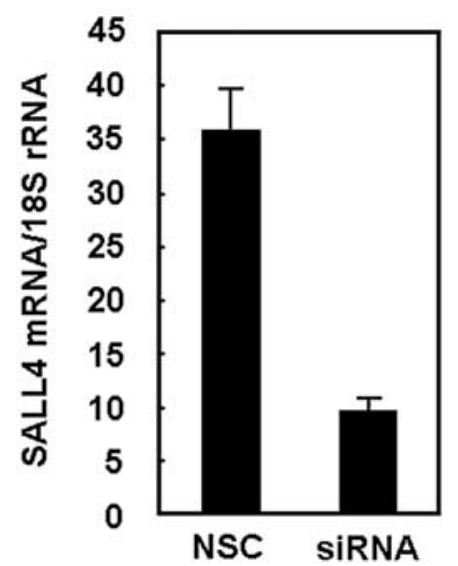

B

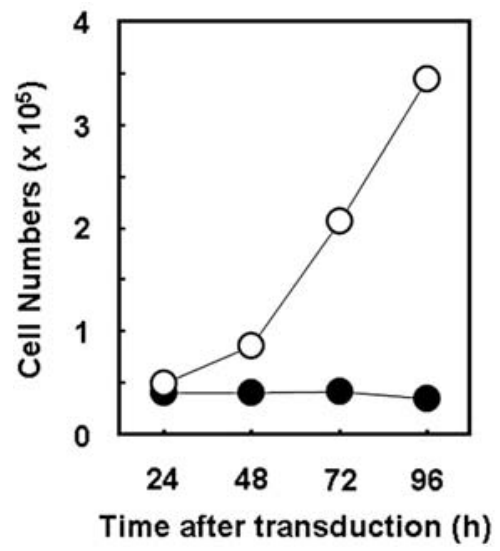

C
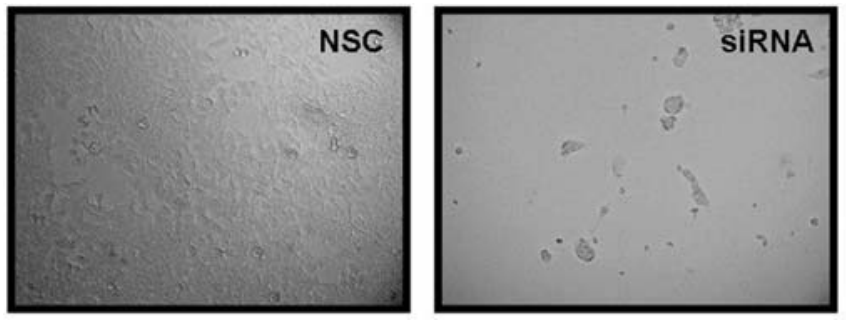

Figure 5. (A) Silencing effect on SALL4 mRNA expression by siRNA transduction of MCF7 cells. Cells were transduced with non-silencing control RNA or siRNA, and total RNA was extracted after $48 \mathrm{~h}$ culture. Twenty nanograms of total RNA was used for quantitative measurement of SALL4 mRNA expression by TaqMan RT-PCR. The expression level of SALLA mRNA in the transduced cells is presented relative to that of $18 \mathrm{~S} \mathrm{rRNA}$. The data represent the mean value for triplicate measurements. As most of SD bars are smaller than the diameter of each circle, the SD bars are not shown in the figure. NSC, non-silencing control RNA; siRNA, small inhibitory RNA. (B) Effect of SALL4-targeted siRNA transduction on the proliferation of MCF7 cells. Viable cells were assessed after 24, 48, 72, and 96 h culture by using a hemocytometer. Data represent the mean for 3 independent experiments. Open circles, control cells transduced with NSC RNA; closed circles, cells transduced with $2.0 \mu \mathrm{g}$ SALL4 siRNA. (C) Microscopic assessment. Representative areas in the well of a 6-well culture plate were photographed under a microscope. Cells were photographed using gray scale. Original magnification x 200 .

$48 \mathrm{~h}$ (Fig. 5A). Thereafter, we determined how the transduction of SALL4 siRNA affects the number of cells. We observed a slight inhibition in growth at $24 \mathrm{~h}$ after transduction. Furthermore, obvious inhibitory effects on cell numbers were observed in siRNA-transduced cells at 48, 72, and $96 \mathrm{~h}$ 

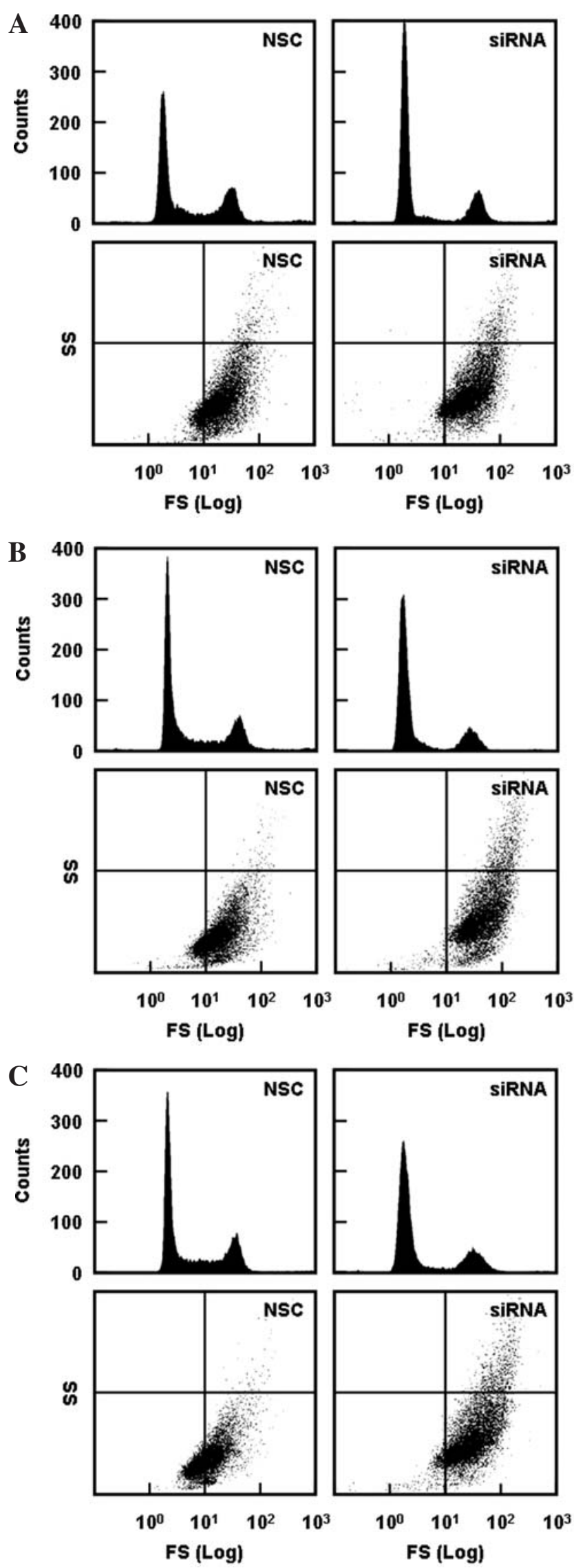

Figure 6. Flow cytometry analysis at 48 h (A), 72 h (B), and 96 h (C) after transduction of SALL4 siRNA into MCF7 cells. Cells were stained with propidium iodide (PI) and subjected to FACS analysis. Cells identical to those stained with PI were analyzed for cytogram distribution determined by forward scatter (FS) and side scatter (SS). The data in the cytogram were obtained using the same gate as that used in the cell cycle analysis. after transduction (Fig. 5B). During the same periods, the cells transduced with non-silencing control (NSC) RNA increased in number without an obvious loss of viability, as determined by microscopic observation. The growth inhibition rate was over $100 \%$ at the examined time-points (20, 19, and $32 \%$ decrease in cell numbers compared to NSC-transduced cells at 48, 72, and 96 h, respectively). After $96 \mathrm{~h}$ of transduction, there were few viable and normally dividing cells, and only relatively larger cell bodies and cell debris were observed (Fig. 5C). As for this effect of siRNA, remarkable growth inhibition was observed also in T47D cells, but more potent effect was shown in MCF7 cells (data not shown).

We then examined how the cell cycle populations were altered during the growth inhibition induced by the transduction of SALL4 siRNA in MCF7 cells. The populations in the sub-G1 phase were not increased at any time-point after transduction. The siRNA-transduced cells showed increased numbers in the G1 phase (47.0-64.5\%) and decreased numbers in the S-phase (20.8-9.1\%) after $48 \mathrm{~h}$, demonstrating typical cell cycle arrest at the G1 phase (Fig. 6A); no alteration was seen in the NSC siRNA-transduced cells. In addition to this phase profile, the overall cell distribution, as determined by 2 parameters (forward scatter, an indicator of cell size; and side scatter, an indicator of intracellular content), was observed. At $48 \mathrm{~h}$, the cells transduced with siRNA showed a slight shift to the upper right (Fig. 6A). At 72 and $96 \mathrm{~h}$ after transduction of SALL4 siRNA, we observed an increase in the number of cells in the G1 phase (72 h, 49.8-62.2\%; $96 \mathrm{~h}, 48.2-59.4 \%)$ and a decrease in the number of cells in the S-phase $(72 \mathrm{~h}$, $22.5-11.1 \%$; $96 \mathrm{~h}, 26.2-12.2 \%)$ with a slight increase in the number of cells entering the early S-phase. During these periods, the cells did not enter into the full range of the S-phase, and the overall evaluation was that the cell cycle was arrested at the G1 phase accompanied by an arrest at the early S-phase. At 72 and $96 \mathrm{~h}$ after transduction, the cell distribution further shifted to the upper right, and this tendency was more obvious at $96 \mathrm{~h}$, consistent with the inhibition of cell division.

\section{Discussion}

This study investigated whether SALL4 mRNA could be used as a marker for the diagnosis of breast cancer. Many studies have analyzed the expression levels of certain oncogenes that are considered to be candidate markers for cancer diagnosis, but the clinical utility of these genes was limited because their expression levels in non-cancer tissues were not negligible. In the present study, we have demonstrated for the first time that SALL4 mRNA expression can be detected with a high sensitivity and specificity in breast cancer tissues.

There have been no previous studies comparing the expression profile of other ES-related molecules (i.e., Nanog, Oct3/4, Sox2, etc.) vs. SALL4 in human malignant tissues. However, interestingly, a recent study clarified the reciprocal regulation of gene expression between SALL4 and Nanog in mouse ES cells (27). As for this relationship, we recently examined the expression of both molecules in various tissues, and found that the expression of Nanog in cancer cells is not significantly increased compared to that in non-cancer cells in human breast cancer patients (28). Consequently, at present, 
no significant correlation between SALL4 and Nanog is speculated. In contrast, Nanog was highly expressed in lung cancers and there was a weak but significant correlation in the expression between both molecules (data not shown). Although future analysis using large numbers of samples remains to be performed, there is a possibility that the expression profile patterns of each ES-related molecule could differ among various organs.

In ROC analysis, the cutoff value of 0.019 was associated with a minimum difference between sensitivity and specificity. An important finding of our study was that SALL4 mRNA was expressed even in the early stages of cancer. Thus, we speculate that the quantitative determination of SALLA mRNA expression would be of clinical value in the diagnosis of small and suspected neoplastic lesions in the breast. In such cases, quantitative RT-PCR is useful when there are only a small number of cells per sample, as obtained by needle or aspiration biopsies. Therefore, to avoid inappropriate therapy in patients without cancer, the cutoff value should ideally be selected so that a high specificity is obtained. In most of the previous studies, the cutoff value was set as the mean +2 SDs of the expression levels observed in non-cancer tissues. In this study, the cutoff value close to the mean +2 SDs of the expression level in non-cancer tissues was 0.031 , and it resulted in a high specificity $(95.0 \%)$ and an allowable sensitivity $(67.4 \%)$. Thus, for clinical application, it is recommended that the cutoff value be set at least around the mean +2 SDs of the expression level in non-cancer tissues.

For the actual clinical use of SALL4, the detection of mRNA or protein expression is theoretically applicable. Recently, one study demonstrated the SALL4 protein expression profiles in cancer and non-cancer breast tissues using immunohistochemistry by their original antibody (29). In their study only 5 of 20 breast cancer specimens showed detectable SALL4 expression, resulting in low sensitivity, although the aims were not to examine the usefulness of SALL4 as a diagnostic marker. Moreover, using large numbers of samples, Cao et al, recently demonstrated similar result that only 10 (esophageal, gastric, and colonic cancers) of 170 metastatic non-germ cell tumors including breast cancers samples showed weak SALL4 protein expression but most of germ cell tumors showed high expression (25). From these facts and our results in this study, at least for breast cancer, SALL4 mRNA detected by highly sensitive RT-PCR might be a good marker to support diagnosis.

In this study, siRNA experiments revealed that the constitutive expression of SALL4 in cancer cells is indispensable for their proliferation. Although the speculated effects of siRNA on the differentiation state could not be observed because of the rapid and potent growth inhibition, our data in human cancer cells are not inconsistent with the observation that the overexpression of the SALL4 gene promotes tumorigenesis of hematopoietic cells in mice (18). However, it is still unclear whether the high expression of SALL4 mRNA causes carcinogenesis or whether carcinogenesis leads to the elevation of SALL4 mRNA expression. Regarding this aspect, Dimri et al reported that the overexpression of the Bmi-1 gene, an oncogene induced by SALL4, in human mammary epithelial cells resulted in cell transformation via the induction of telomerase activity (22). In addition, our recent study showed that Bmi-1 mRNA was also highly expressed in the early clinical stages of breast cancer (30). These two evidences suggest that up-regulation of SALL4 causes carcinogenesis in breast cancer. Furthermore, in the current study, siRNA against SALL4 completely inhibited cell growth and caused cell cycle arrest at G1 and the early S-phase. Our results are consistent with the observation that Bmi-1 downregulates INK4a/ARF (23), thereby leading to uncontrollable cell cycle transition from the G1 to the S-phase. Taken together, our data and the other evidence suggest that SALL4 could exert its oncogenic potential in human malignancies, especially in the development of breast cancer via the induction of Bmi-1. Thus, the evidence strongly supports the significance of the measurement of SALL4 mRNA as the diagnostic tool of breast cancer. In addition, SALL4 may represent a novel target for cancer therapy, although the effect on siRNA in non-cancer cells is still unclarified.

\section{References}

1. Benson JR, Jatoi I, Keisch M, Esteva FJ, Makris A and Jordan VC: Early breast cancer (review). Lancet 373: 1463-1479, 2009.

2. Koshida S, Kobayashi D, Moriai R, Tsuji N and Watanabe N: Specific overexpression of OLFM4(GW112/HGC-1) mRNA in colon, breast and lung cancer tissues detected using quantitative analysis. Cancer Sci 98: 315-320, 2007.

3. Wascher RA, Huynh KT, Giuliano AE, Hansen NM, Singer FR, Elashoff D and Hoon DS: Stanniocalcin-1: a novel molecular blood and bone marrow marker for human breast cancer. Clin Cancer Res 9: 1427-1435, 2003.

4. Chen XQ, Bonnefoi H, Pelte MF, Lyautey J, Lederrey C, Movarekhi S, Schaeffer P, Mulcahy HE, Meyer P, Stroun M and Anker P: Telomerase RNA as a detection marker in the serum of breast cancer patients. Clin Cancer Res 6: 3823-3826, 2000.

5. Pardal R, Molofsky AV, He S and Morrison SJ: Stem cell selfrenewal and cancer cell proliferation are regulated by common networks that balance the activation of proto-oncogenes and tumor suppressors (review). Cold Spring Harb Symp Quant Biol 70: 177-185, 2005.

6. Yasuda SY, Tsuneyoshi N, Sumi T, Hasegawa K, Tada T, Nakatsuji N and Suemori H: NANOG maintains self-renewal of primate ES cells in the absence of a feeder layer. Genes Cells 11: 1115-1123, 2006.

7. Zhou Q, Chipperfield H, Melton DA and Wong WH: A gene regulatory network in mouse embryonic stem cells. Proc Natl Acad Sci USA 104: 16438-16443, 2007.

8. Zhang J, Tam WL, Tong GQ, Wu Q, Chan HY, Soh BS, Lou Y, Yang J, Ma Y, Chai L, Ng HH, Lufkin T, Robson P and Lim B: Sall4 modulates embryonic stem cell pluripotency and early embryonic development by the transcriptional regulation of Pou5f1. Nat Cell Biol 8: 1114-1123, 2006.

9. Jiang J, Chan YS, Loh YH, Cai J, Tong GQ, Lim CA, Robson P, Zhong S and $\mathrm{Ng} \mathrm{HH}$ : A core Klf circuitry regulates self-renewal of embryonic stem cells. Nat Cell Biol 10: 353-360, 2008.

10. Okita K, Ichisaka T and Yamanaka S: Generation of germlinecompetent induced pluripotent stem cells. Nature 448: 313-317, 2007.

11. Tsubooka N, Ichisaka T, Okita K, Takahashi K, Nakagawa M and Yamanaka S: Roles of Sall4 in the generation of pluripotent stem cells from blastocysts and fibroblasts. Genes Cells 14: 683-694, 2009

12. Yu J, Vodyanik MA, Smuga-Otto K, Antosiewicz-Bourget J, Frane JL, Tian S, Nie J, Jonsdottir GA, Ruotti V, Stewart R, Slukvin II and Thomson JA: Induced pluripotent stem cell lines derived from human somatic cells. Science 318: 1917-1920, 2007.

13. Hyslop L, Stojkovic M, Armstrong L, Walter T, Stojkovic P, Przyborski S, Herbert M, Murdoch A, Strachan T and Lako M: Downregulation of NANOG induces differentiation of human embryonic stem cells to extraembryonic lineages. Stem Cells 23: 1035-1043, 2005. 
14. Sakaki-Yumoto M, Kobayashi C, Sato A, Fujimura S, Matsumoto Y, Takasato M, Kodama T, Aburatani H, Asashima M, Yoshida N and Nishinakamura R: The murine homolog of SALL4, a causative gene in Okihiro syndrome, is essential for embryonic stem cell proliferation, and cooperates with Sall1 in anorectal, heart, brain and kidney development. Development 133: 3005-3013, 2006.

15. Jürgens G: Head and tail development of the Drosophila embryo involves spalt, a novel homeotic gene. EMBO J 7: 189-196, 1988.

16. Al-Baradie R, Yamada K, St Hilaire C, Chan WM, Andrews C, McIntosh N, Nakano M, Martonyi EJ, Raymond WR, Okumura S, Okihiro MM and Engle EC: Duane radial ray syndrome (Okihiro syndrome) maps to 20q13 and results from mutations in SALL4, a new member of the SAL family. Am J Hum Genet 71: 1195-1199, 2002.

17. Kohlhase J, Heinrich M, Liebers M, Fröhlich Archangelo L, Reardon W and Kispert A: Cloning and expression analysis of SALL4, the murine homologue of the gene mutated in Okihiro syndrome. Cytogenet Genome Res 98: 274-277, 2002.

18. Ma Y, Cui W, Yang J, Qu J, Di C, Amin HM, Lai R, Ritz J, Krause DS and Chai L: SALL4, a novel oncogene, is constitutively expressed in human acute myeloid leukemia (AML) and induces AML in transgenic mice. Blood 108: 2726-2735, 2006.

19. Cui W, Kong NR, Ma Y, Amin HM, Lai R and Chai L: Differential expression of the novel oncogene, SALL4, in lymphoma, plasma cell myeloma, and acute lymphoblastic leukemia. Mod Pathol 19: 1585-1592, 2006.

20. Yang J, Chai L, Gao C, Fowles TC, Alipio Z, Dang H, Xu D, Fink LM, Ward DC and Ma Y: SALL4 is a key regulator of survival and apoptosis in human leukemic cells. Blood 112: 805-813, 2008

21. Yang J, Chai L, Liu F, Fink LM, Lin P, Silberstein LE, Amin HM, Ward DC and Ma Y: Bmi-1 is a target gene for SALL4 in hematopoietic and leukemic cells. Proc Natl Acad Sci USA 104: 10494-10499, 2007.
22. Dimri GP, Martinez JL and Jacobs JJ, et al: The Bmi-1 oncogene induces telomerase activity and immortalizes human mammary epithelial cells. Cancer Res 62: 4736-4745, 2002.

23. Liu L, Andrews LG and Tollefsbol TO: Loss of the human polycomb group protein BMI1 promotes cancer-specific cell death. Oncogene 25: 4370-4375, 2006.

24. Cao D, Li J, Guo CC, Allan RW and Humphrey PA: SALL4 is a novel diagnostic marker for testicular germ cell tumors. Am J Surg Pathol 33: 1065-1077, 2009

25. Cao D, Humphrey PA and Allan RW: SALL4 is a novel sensitive and specific marker for metastatic germ cell tumors, with particular utility in detection of metastatic yolk sac tumors. Cancer 115: 2640-2651, 2009.

26. Cao D, Guo S, Allan RW, Molberg KH and Peng Y: SALL4 is a novel sensitive and specific marker of ovarian primitive germ cell tumors and is particularly useful in distinguishing yolk sac tumor from clear cell carcinoma. Am J Surg Pathol 33: 894-904, 2009.

27. Wu Q, Chen X, Zhang J, Loh YH, Low TY, Zhang W, Zhang W Sze SK, Lim B and Ng HH: Sall4 interacts with Nanog and co-occupies Nanog genomic sites in embryonic stem cells. J Biol Chem 281: 24090-24094, 2006.

28. Nirasawa S, Kobayashi D, Tsuji N, Kuribayashi K and Watanabe N: Diagnostic relevance of overexpressed Nanog gene in early lung cancers. Oncol Rep 22: 587-591, 2009.

29. Bard JD, Gelebart P, Amin HM, Young LC, Ma Y and Lai R: Signal transducer and activator of transcription 3 is a transcriptional factor regulating the gene expression of SALL4. FASEB J 23: 1405-1414, 2009

30. Saeki M, Kobayashi D, Tsuji N, Kuribayashi K and Watanabe N: Diagnostic importance of overexpression of Bmi-1 mRNA in early breast cancers. Int J Oncol 35: 511-515, 2009. 